\title{
MUJERES LATINAS PROFESIONALES Y MIGRANTES CASOS DE EXITO DE MUJERES CALIFICADAS EN EL SUR DE LA FLORIDA, EE.UU.
}

PROFESSIONAL MIGRANT LATIN AMERICAN WOMEN SUCCESS STORIES OF SKILLED WOMEN IN SOUTH FLORIDA, USA.

\author{
MARITZA FIGUEROA* \\ Recibido 5 de julio del 2016 \\ Aceptado 2 de septiembre del 2016
}

* Pontificia Universidad Católica del Ecuador, Quito, Ecuador (mfiguero818@puce.edu.ec) 



\section{MUJERES LATINAS PROFESIONALES Y MIGRANTES \\ CASOS DE EXITO DE MUJERES \\ CALIFICADAS EN EL SUR DE LA FLORIDA, EE.UU.}

\section{PROFESSIONAL MIGRANT LATIN AMERICAN WOMEN SUCCESS STORIES OF SKILLED WOMEN IN SOUTH FLORIDA, USA.}

MARITZA FIGUEROA

palabras clave: Género, migración calificada, interseccionalidad, empleo

key words: Gender, skilled migration, intersectionality, employment

\section{RESUMEN}

Con el propósito de conocer nizaciones con sedes tanto en América como mujeres profesionales desarrollaLatina como en Estados Unidos, se les ron sus carreras en un país diferente de su país de origen, se realizó un estudio exploratorio para responder varias interrogantes sobre la trayectoria, vida profesional y personal de veintitres mujeres latinas, quienes poseen títulos universitarios y que residen al sur del Estado de la Florida, en los Estados Unidos de América. Ellas al formar parte de orgahace posible no solo experimentar satisfacción en su trabajo, sino que también pudieron establecerse en el extranjero con sus familias de manera exitosa. Este artículo examina también, aspectos de interseccionalidad relacionados con género, clase y etnia, para así entender dichas historias de migración calificada y generar insumos al estudio de procesos 
migratorios de mujeres profesionales y de origen latinoamericano que forman parte del mercado laboral de los Estados Unidos de América.

\section{ABSTRACT}

In an attempt to learn how professional women have developed their careers in a country different from their country of birth, an exploratory study has been conducted highlighting lives of twenty-three professional Latinas living in South Florida who are experiencing both a high degree of job satisfaction and a positive overall migrant experience, following their journey with organizations that have enabled them to venture abroad and establish their lives in the United States of America. In addition, this article examines aspects of intersectionality with respect to gender, class, and ethnicity in order to better understand the origins of successful skilled labor migration and provide more elements on how professional female migrants of South American origin are employed in the United States of America.

\section{INTRODUCCIÓN}

El crecimiento sin precedentes de la migración calificada y la necesidad de entender las relaciones de género dentro de las tendencias globales; por medio de este estudio, se conocerán las realidades que experimentaron 23 mujeres de diferentes países de América Latina que residen en el estado de la Florida, uno de los cinco Estados con mayor población latina (22,5\%) en los Estados Unidos de América, según el último censo que se realizó en dicho país en el año 2010. Se hace una revisión teórica de los procesos migratorios de personas calificadas, su relación e importancia al considerar aspectos de género en procesos migrato- rios y posteriormente se analizan las realidades de estas latinas, quienes poseen estudios universitarios a nivel de pre y posgrado. Finalmente, se conocerá cuáles fueron los patrones de migración de estas mujeres profesionales, sus trayectorias de desplazamiento y los sectores en los que laboran.

Tal como lo expresa Koffman (2012) en su estudio de género y migración calificada en Europa, existen pocos estudios que se centren en la composición de género, experiencias y obstáculos para movilidad de migrantes calificadas; por lo tanto, este estudio examina aspectos de interseccionalidad que se 
relacionan con género, etnia y clase, así como aspectos de descalificación en relación con sus contrapartes masculinos, también residentes en el Estado de la Florida. Finalmente, se dan a conocer algunas de las razones de éxito de estas profesionales de origen latinoamericano en los Estados Unidos de América, con lo que se contribuye al estudio de los movimientos internacionales de mujeres calificadas, los cuales se incrementan a nivel mundial.

\section{Migración Calificada y Migración Femenina}

Para propósito de este estudio, se adoptó el concepto de "migrante calificado" definido por Lowell, Lindlay y Steward (2004) y citado en Lozano y Gandini (2009), donde migrantes calificados son: "aquellas personas con educación terciaria o possecundaria, quienes buscan oportunidades laborales y de vida, en un país distinto al de su país de origen". Para entender el fenómeno de la migración calificada, es necesario, tal como lo expresan Lozano y Gandini (2009), considerar que este fenómeno evoluciona de posturas extremas y que posee una descripción variada en cuanto a su definición durante los diversos procesos migratorios mundiales.

Es así que, en su ponencia presentada en 2011 en Quito, estos dos autores (Lozano y Gandini) expresan que dentro de los estudios de migración calificada existen, dos posturas totalmente opuestas que tratan de explicar este proceso migratorio de individuos con educación terciaria o possecundaria. Así, la primera postura es la visión negativa de los años sesenta y setenta que consideraba a la migración calificada como una fuga de cerebros, donde personas con un alto perfil educativo y profesional optaban por dejar sus países de origen generalmente en vías de desarrollo y buscaban oportunidades laborales en otro país industrializado o desarrollado, los cuales de alguna manera ofrecían a los migrantes mejores salarios y oportunidades laborales debido a su educación y experiencia. La segunda postura, un poco más positiva de los años noventa, es la que consideraba a este movimiento de personas como un proceso impulsor del desarrollo, donde gracias a la globalización mundial, tanto países emisores de emigrantes como los países receptores se beneficiaban ya sea los primeros al recibir remesas económicas generadas por los emigrantes profesionales y los segundos se favorecerían del intercambio de conocimientos y experiencias que podrían aportar los migrantes en el país de destino. (Lozano, et al. (2011).

Al considerar los movimientos internacionales de la población calificada, es necesario tomar en cuenta que dichos procesos migratorios son complejos e 
involucran diversos factores. Las redes de conexión de los migrantes, las características de los países emisores y receptores y los mercados laborales en los cuales se emplean dichos profesionales, son elementos que no solo convierten a la migración calificada en un proceso social sostenido, sino que, por su naturaleza, abarcan a otros actores. Por ejemplo, los familiares del migrante principal se involucran voluntaria o involuntariamente en este proceso, ya que muchas veces el traslado a otro país no solo comprende a una persona sino a familias enteras. Así también, estos movimientos de individuos suelen ser el resultado de acciones gubernamentales, tanto en los países de origen como de destino, donde se crean mecanismos legales que favorecen a la migración de personal calificado y que de alguna manera participan e influencian el proceso. Finalmente, las acciones de actores económicos privados en países receptores, ya sean instituciones con o sin fines de lucro, son los entes que fomentan la migración calificada y participan activamente en ella, al ser los empleadores directos de profesionales en destino. ((Lozano, F. et al. (2011).

\section{¿Por qué es importante analizar a la migración calificada que viaja a Estados Unidos?}

En comparación con otras regiones del mundo, América Latina y el
Caribe experimentaron un incremento del $91 \%$ de los movimientos de recursos humanos calificados hacia los Estados Unidos de América entre los años 1990 y 2000, donde el 35\% de migrantes posee altos niveles de educación, tales como maestrías y doctorados. Dado que la migración calificada manifiesta un incremento acelerado y constante entre los años 1999 y 2009, representa sin duda un componente importante dentro de los movimientos migratorios a nivel mundial. Consecuentemente, estas salidas de personas involucran a más del 10\% de la población de origen, lo que implica un grupo claro de interés. Además, es importante conocer cuál es el rol de género dentro de este grupo de migrantes, ya que tal como lo manifiestan Lozano y Gandini (2009), es necesario realizar un análisis detallado de acuerdo con el sexo de los migrantes para establecer si el factor género es un determinante en los procesos migratorios.

\section{¿Por qué es importante examinar a la migración calificada femenina?}

En su estudio de migrantes calificados en Estados Unidos, Soledad Coloma (2012) demuestra una creciente participación de mujeres migrantes calificadas. Por ejemplo, existe un 24\% de mujeres emigrantes ecuatorianas residentes en ese país, quienes cuentan con estudios superiores a diferencia del 17\% 
de hombres con similar educación. Así también, la autora destaca que hay más mujeres migrantes $(26,8 \%)$ con un alto grado de educación (educación superior) que viven en los Estados Unidos de América, en comparación del 18,2\% de hombres migrantes, siendo los Estados Unidos un destino particular de la migración calificada femenina.

Al examinar la migración calificada como un proceso social sostenido, es importante considerar además la postura de Riaño y Baghdadi (2007) quienes expresan que el entendimiento sobre el fenómeno de la migración calificada aún permanece limitado debido a cuatro razones:

- La primera se debe a que la bibliografía sobre migración calificada analiza al fenómeno de la migración como un hecho netamente masculino; por lo tanto, se ignora implícitamente el análisis de datos de mujeres migrantes calificadas.

- La segunda razón establece que la participación poco significativa de las mujeres en los mercados laborales internacionales, no permite hacer una distinción de roles de género, ya que generalmente las mujeres ocupan posiciones marginales en dichos mercados, lo que hace insuficiente expresar un efecto directo en el mercado de trabajo.
- Una tercera razón indica que varias de las investigaciones sobre el tema se centran en migrantes que ingresan a sus países de destino por razones netamente de trabajo y dejan de lado migrantes calificados que ingresan por razones familiares o de refugio.

- Finalmente, las autoras expresan que existe la necesidad de considerar factores de migración relacionados con matrimonios o situaciones de reunificación familiar cuando se habla de migración calificada femenina. Tal como se muestra en el estudio en Suiza, aproximadamente el 43\% de migrantes calificadas dejan sus países de origen por razones ajenas a lo laboral, comparado con el $26 \%$ de migrantes calificadas que ingresan al territorio suizo por razones de trabajo.

De esta manera, dentro de los análisis de movimientos migratorios es necesario ahondar en factores específicos que muestren tendencias y características particulares asociadas a mujeres, para así determinar directrices que se relacionan con el tema de la migración de recursos humanos calificados. Si se toma la mirada de interseccionalidad y se examinan características individuales dentro de flujos de migración calificada, fueron estos flujos procesos condiciona- 
dos y estructurados por agentes o mecanismos externos que de alguna manera promueven y retroalimenten este fenómeno, se podrá conocer sí el tiempo de vinculación con el empleador y el hecho de examinar a mujeres profesionales, en este caso de origen Latinoamericano, determinan su ingreso y estadía permanente en los Estados Unidos de América.

\section{Descualificación}

Al estudiar la población migrante calificada, un hecho común que experimentan dichos migrantes es la descualificación. Koffman (2012) manifiesta que existen dos consecuencias directas asociadas a este fenómeno. La una relacionada con el desempeño laboral y la otra relacionada con la ejecución del trabajo en sí. El primer efecto de descualificación ocurre cuando migrantes calificados se desempeñan en áreas totalmente diferentes al campo de formación original. El segundo efecto ocurre cuando dichos migrantes ejecutan tareas cuyos requerimientos demandan un nivel menor de parámetros de los que estos poseen. Por lo tanto, estos dos aspectos están directamente relacionados con la noción que existe un desaprovechamiento de conocimientos, habilidades y destrezas de los migrantes con educación de alto nivel. Es así que dichos profesionales en destino desempeñarán actividades por debajo de sus capacidades o a su vez la- borarán en áreas ajenas a su educación original; este hecho es un desperdicio de talentos o brain waste (Bauer, 2003). Al hacer referencia al por qué de estos procesos de descualificación, es importante analizar la participación de mujeres migrantes profesionales en el mercado laboral en destino. A base de los datos de estudios de mujeres calificadas realizado por Dumont y Leibig (2005) en países miembros de la Organización para la Cooperación y el Desarrollo Económicos (OECD - por sus siglas en inglés) y citado por Koffman (2012), dichas migrantes tendrían más probabilidades de estar sobre calificadas para desempeñar sus empleos que en contraste con hombres profesionales también migrantes.

Por esta razón, para determinar si existe una descualificación de mujeres calificadas, se cree pertinente conocer su participación dentro del mercado laboral. Tal como lo mencionan Riaño y Baghdadi (2007), se da muy poca atención a la correlación entre clase, etnia y género en los estudios de migración calificada: por lo tanto, para generar un mejor entendimiento sobre la inserción laboral de migrantes calificados es necesario considerar la interseccionalidad de estos tres factores, a más de establecer la posición o rango que una persona ocupa en su lugar de trabajo y la participación activa o no en los mercados laborales en destino. 
Revista PUCE. ISSN 1390-7719. Núm.103. 3 de mayo de 2016 -

\section{DESCRIPCIÓN DEL ESTUDIO Y METODOLOGÍA}

\section{Características generales de la población del estudio}

Este estudio exploratorio de caso se realizó entre los meses de julio y agosto del año 2014 por medio de una encuesta virtual, la cual se aplicó a una población migrante de mujeres calificadas de origen latinoamericano que residían desde el año 2004 hasta el año 2014 en el sur del Estado de la Florida en los Estados Unidos de América. La muestra de este estudio la conformaron 23 mujeres de 8 países latinoamericanos (Argentina, Brasil, Chile, Colombia, Ecuador, México, Puerto Rico y Venezuela). Adicionalmente, se hicieron entrevistas de seguimiento en línea con ciertos casos representativos para el estudio; por ejemplo, mujeres latinas de más de 25 años de edad con estudios de tercer nivel (superiores) cursados en los Estados Unidos.

Los rangos de edad de este grupo se constituyen de la siguiente forma: participantes entre 25 a 35 años (39\%), participantes de 36 a 46 años (39\%) y participantes de 47 años o más (22\%), edades que corresponden a la población económicamente activa. Respecto al nivel de educación, todas las encuestadas poseen un título de tercer nivel, siendo la mayoría (34,8\%) mujeres que concluyeron sus estudios universitarios a nivel de licenciatura o ingeniería en el respectivo país de origen. Dentro de los porcentajes significativos están las profesionales tituladas en sus países de origen a nivel de maestría o MBAs (Master of Business Administration - por sus siglas en inglés) con el 17,4\%, así como las profesionales que recibieron su titulación en un nivel similar de maestría o MBAs, pero en destino con un igual porcentaje (17,4\%). El siguiente grupo representativo incorpora a las migrantes a nivel de licenciatura quienes obtuvieron su título en los Estados Unidos de América (13\% del total). Para más detalles de cada nivel educativo del total de las participantes del estudio, ver Anexo 3.

\section{Inserción en el mercado laboral de los Estados Unidos}

Para abordar las trayectorias laborales de este grupo, es importante analizar su inserción y participación en el mercado laboral en destino. Siete son las áreas de trabajo en las cuales se encuentran empleadas las profesionales latinas de este estudio. Así, las áreas con porcentajes preponderantes son: Finanzas,

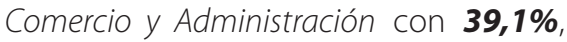
Información Tecnológica y Telecomunicaciones al igual que el área de Ciencias Sociales y del Comportamiento con $\mathbf{1 7 , 4 \%}$. Las profesionales que laboran en áreas de Salud, Medicina, Trabajo Social, ejem- 
plifican el $8.7 \%$ de la población y finalmente el porcentaje de profesionales sin empleo al momento de correr la encuesta representaban el 8,7\%.

Dado que existe un $8.7 \%$ de migrantes empleadas en áreas de la salud, medicina y trabajo social, de alguna manera estas cifras ratifican la tendencia histórica de empleo femenino, donde mujeres migrantes generalmente encuentran un nicho de mercado para ser contrata- das. A pesar que la gran mayoría se desempeña en áreas de comercio, finanzas y administración tal como lo muestra la Tabla 1, un total del 30,4\% de participantes trabaja en áreas del bienestar (Educación / Actividades sin fines de lucro, salud, medicina, trabajo social, ciencias sociales y comportamiento) tomando en cuenta que dentro del área de ciencias del comportamiento se incluyen posiciones del área de Personal y Recursos Humanos.

TABLA 1

ÁREAS Y SECTORES DE PARTICIPACIÓN EN EL

MERCADO LABORAL

\begin{tabular}{ccc}
\hline ÁREA / SECTOR & PORCENTAJE & TOTAL \\
\hline Finanzas, comercio y administración & $39,1 \%$ & 9 \\
\hline Información tecnológica, telecomunicaciones & $17,4 \%$ & 4 \\
\hline Medios, entretenimiento & $4,3 \%$ & 1 \\
\hline Educación/act. sin fines de lucro & $4,3 \%$ & 1 \\
\hline Salud, medicina, trabajo social & $8,7 \%$ & 2 \\
\hline Ciencias sociales y comportamiento (incl. RRHH) & $17,4 \%$ & 4 \\
\hline Desempleada & $8,7 \%$ & 2 \\
\hline Otro (transporte/logística) & & 1 \\
\hline
\end{tabular}

Fuente: Encuesta Migración Calificada de Mujeres Latinas en el sur de la Florida, USA, 2014 Elaborado por: Maritza Figueroa

Al analizar la inserción laboral desde una perspectiva de género, es importante examinar su concentración en el área financiera, la cual es parte de las áreas relacionadas con la industria de conocimiento, la misma que generalmente está dominada por hombres (Koffman, 2012). A base de los datos obtenidos el área de Finanzas, Comercio y Administra- ción, se muestra como el área de mayor concentración laboral de este grupo de estudio. Razón por la cual se consultó a tres de las participantes, por medio de entrevistas a profundidad y comunicación electrónica, sobre su proceso de inserción laboral en Estados Unidos y los cargos de trabajo que desempeñan. Ellas expresaron ser Analista Financiera, 
Vendedora especializada en productos de logística y Directora de Finanzas/Billing, puestos que ya desempeñaban en origen o que poseían experiencia previa antes de ser ascendidas o contratadas en la posición actual. Dado que este aspecto difiere de las aseveraciones sobre migración calificada, que indica que las mujeres migrantes profesionales tienen muy poca participación en el mercado laboral, especialmente en áreas del conocimiento (Koffman, 2012), en este estudio se demuestra que son la mayoría (39\%) de la población encuestada. Se podría considerar a este hallazgo como un área que demandaría una mayor investigación y análisis en futuros estudios sobre migración calificada femenina.

Otro aspecto a considerar dentro del análisis de inserción laboral es conocer sobre la participación activa en el mercado laboral de la población examinada. Cabe destacar que a excepción de dos participantes (9\%), el resto de encuestadas posee un empleo. Además, para determinar si experimentan procesos de descualificación, se consultó al grupo si realizaban actividades acordes con sus estudios. Los resultados de la Tabla 2 indican que el $61 \%$ de estas migrantes participan activamente del mercado de trabajo y sus tareas y responsabilidades están relacionadas con sus niveles formativos y habilidades. Así también se encontró que el 17\% de participantes se desempeñan en tareas que exigen un título universitario pero dichas tareas no se relacionan con su formación universitaria original.

TABLA 2

ACTIVIDAD LABORAL RELACIONADA CON

ESTUDIOS UNIVERSITARIOS

\begin{tabular}{ccc}
\hline TIPO DE RELACIÓN & PORCENTAJE & TOTAL \\
\hline 100\% relacionado & $60,9 \%$ & 14 \\
\hline Sí - pero empleo de medio tiempo & $4,3 \%$ & 1 \\
\hline No - pero mi trabajo exige un título universitario & $17,4 \%$ & 4 \\
\hline No - mi empleo actual no requiere estudios universitarios & $8,7 \%$ & 2 \\
\hline No - soy ama de casa o estudiante & $8,7 \%$ & 2 \\
\hline
\end{tabular}

Fuente: Encuesta Migración Calificada de Mujeres Latinas en el sur de la Florida, USA, 2014

Elaborado por: Maritza Figueroa

Si se toma en cuenta que más de la mitad de mujeres encuestadas no experimentan descualificación y que la gran mayoría posee un empleo que demanda educación superior, se puede concluir que existen ciertas diferencias 
con lo que se demuestra en la literatura sobre migración calificada (Lozano y Gandini, 2009; Koffman, 2012), donde se afirma que mujeres migrantes profesionales experimentan grandes porcentajes de descualificación. Por esta razón se hace necesario analizar en detalle y con una perspectiva de interseccionalidad las hipótesis, que posiblemente requieran un mayor sustento para afirmar que las mujeres profesionales y migrantes experimentan formas de desaprovechamiento de sus conocimientos, habilidades y destrezas en destino, tema que demanda una mayor atención y se debería profundizar a futuro.

\section{Trayectoria laboral de mujeres latinas calificadas en el sur de la Florida}

Para conocer las trayectorias de migración de este grupo, se examinó en primera instancia la razón de viaje a los Estados Unidos, donde la mayoría (11) respondió a razones laborales que corresponde casi al 50\%. Las mujeres que ingresaron por razones de matrimonio representan un 22\%, por estudios un $18 \%$ y por reunificación familiar un 13\%; estos resultados ejemplifican lo que Lozano y Gandini, (2011) mencionaron en su ponencia en Quito, referente a los flujos de migración calificada, los cuales están altamente estructurados por agentes y mecanismos que promueven y re- troalimentan el fenómeno migratorio y que exceden las fronteras del Estado nacional. En este sentido, se entrevistó con una de las participantes para conocer qué tipo de organizaciones generaron su salida del país de origen y expresó: "buscaba una experiencia internacional y por eso decidí trabajar en una empresa multinacional. Estaba convencida de mis capacidades y después de un par de años trabajando con ellos, pude aplicar a un puesto en Estados Unidos y por eso migré" (M. Figueroa, comunicación personal con Clara', agosto 9, 2014).

Respecto al tiempo de residencia en EE.UU. como lo muestra la Tabla 3, casi la mitad de la población de mujeres encuestadas vivió en destino por más de 11 años, lo que es un indicador de su éxito laboral tal como lo mencionan Lozano y Gandini (2009), donde el tiempo de residencia es un factor favorable para la inserción laboral. Al hacer una comparación con el tipo de residencia que poseen las participantes se demuestra que el $44 \%$ posee ciudadanía americana y el 30\% posee residencia permanente (para más detalles ver Anexo 4), lo cual implicaría que las participantes lograron acceder y asegurar sus formas de permanencia en destino de manera estable y sin límite de tiempo.

1 Nombre de la participante es diferente por razones de confidencialidad 
ReVista PUCE. ISSN 1390-7719. Núm, 103. 3 de MAYO de 2016 3 De noviembre de 2016. Maritza Figueroa, PP. 253-272

TABLA 3

TIEMPO DE RESIDENCIA EN DESTINO

\begin{tabular}{ccc}
\hline TIEMPO & PORCENTAJE & TOTAL \\
\hline Menos de 1 año & $4,3 \%$ & 1 \\
\hline De 2 a 5 años & $21,7 \%$ & 5 \\
\hline De 6 a 10 años & $26,1 \%$ & 6 \\
\hline 11 años o más & $47,8 \%$ & 11 \\
\hline
\end{tabular}

Fuente: Encuesta Migración Calificada de Mujeres Latinas en el sur de la Florida, USA, 2014

Elaborado por: Maritza Figueroa

Para entender si la trayectoria migratoria de este grupo se debió a relaciones netamente laborales, se examinó el tiempo de vinculación con el empleador actual, ya que la mayoría de encuestadas ingresó en los Estados Unidos de América por trabajo. Coloma (2012) menciona que existen mecanismos que se utilizan en los Estados Unidos para atraer a trabajadores calificados. Uno de ellos consiste en visas de trabajo temporales con una duración de tres años que pueden ser extendidas por tres años más; además tal como lo manifiesta Conolly (2010) citado en Coloma (2012), si las personas poseen un visado de trabajo temporal tipo $\mathrm{H} 1$-B se les permite solicitar residencia permanente, siempre y cuando los empleadores den fe que trabajadores nativos no sean afectados por su contratación. A base de los resultados del gráfico 1 se demuestra que un tercio de las participantes permanece vinculado con el mismo empleador por más de 7 años (35\%), lo cual implicaría que uno de los mecanismos de estabilización en destino es el de visado y residencia descrito anteriormente.

\section{GRÁFICO 1}

TIEMPO DE VINCULACIÓN CON EMPLEADOR ACTUAL

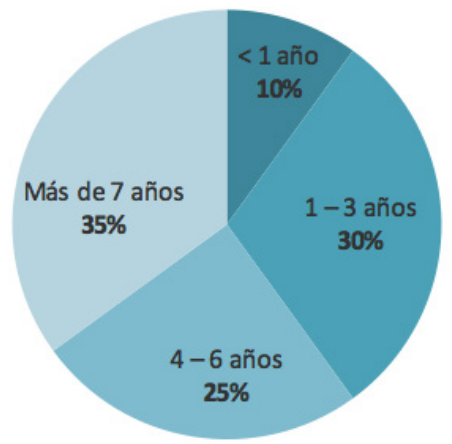

Fuente: Encuesta Migración Calificada de Mujeres Latinas en el sur de la Florida, USA, 2014 Elaborado por: Maritza Figueroa

Para analizar la trayectoria migratoria, se consultó a las participantes sobre sus asentamientos previos a la entrada a los Estados Unidos de América. Cuatro de ellas (17\%) vivían en un territorio distinto de su país de origen y Europa es el continente donde la mayoría de profesionales de este grupo residía y tan solo una participante de este $17 \%$ residía en el continente asiático. Al consultar sobre si dichas encuestadas tenían algún interés de cambiar su residencia actual motivada por razones laborales, con condiciones de trabajo mejores o similares a las vigentes, un 39\% manifestó que 
sí tendrían interés en continuar con una trayectoria migratoria en otro Estado de los Estados Unidos de América y un 35\% desearía explorar otra región del mundo, lo cual implica que más de la mitad de este grupo de mujeres desean seguir su carrera profesional fuera de su país de origen. Para más información sobre destinos y rutas migratorias, ver anexo 5 .

\section{Factores que influyen en las rutas migratorias}

Para abordar las trayectorias laborales, se examinó el nivel educativo, la razón de ingreso en los Estados Unidos de América y la relación entre el país de origen y de destino mediada por el organismo empleador de la migrante. Dado que los resultados arrojan información diferente a lo que se expresa en la literatura de migración calificada y migración calificada femenina, es importante analizar la interrelación de clase, etnia y género de esta población, la cual juega un rol importante al modelar la posición individual de mujeres en el mercado laboral, tal como lo demuestran Riaño y Baghdadi (2007).

Es así que se consultó sobre otras características concernientes a la categoría de recursos culturales y sociales. Los resultados mostraron que 8 de las participantes (35\%) hablan una lengua adicional al idioma inglés, casi un tercio de la población ocupa cargos gerencia- les, mientras que el otro tercio se desempeña en roles a nivel administrativo y operativo tal como lo indica el Gráfico 2. Ejemplos de estos cargos descritos en la encuesta son: analista financiera senior, dueña de negocio propio, gerente de su propia empresa.

\section{GRÁFICO 2}

TIPO DE POSICIÓN QUE REALIZA LA ENCUESTADA

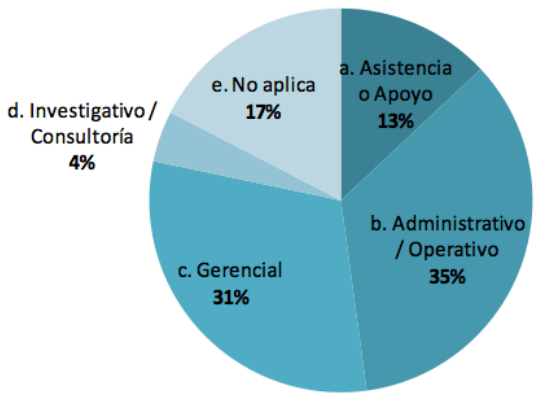

Fuente: Encuesta Migración Calificada de Mujeres Latinas en el sur de la Florida, USA, 2014

Elaborado por: Maritza Figueroa

En relación con el estado civil, la mayoría de participantes están casadas (74\%) y la mitad tiene hijos (52\%). Es decir que este es un grupo de mujeres que logró combinar sus relaciones laborales con su vida familiar. Para conocer más sobre este tema se consultó sobre qué aspectos fueron descuidados por el éxito laboral migratorio. Las participantes mencionaron en su mayoría (58\%) sacrificar el aspecto familia, es decir el cuidado de hijos, padres, hermanos o realizar actividades con miembros del núcleo 
familiar, tal como lo demuestra el Gráfico 3. Así también, el 37\% manifestó el dejar de lado aspectos de atención a la pareja o el contraer matrimonio y el 32\% indicó el no dar prioridad a su bienestar físico, espiritual y emocional por enfocarse en consolidar su éxito laboral migratorio.

\section{GRÁFICO 3}

ASPECTOS COMPROMETIDOS POR EL ÉXITO LABORAL MIGRATORIO

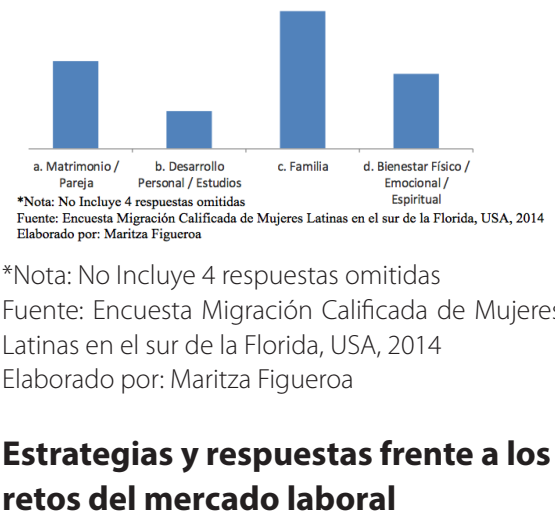

Este grupo demostró poseer características especiales como mujeres calificadas, no solo por su deseo de continuar con sus rutas migratorias, si así fuera el caso, sino que es un grupo de profesionales que posee posiciones de liderazgo e influencia en las organizaciones en las que laboran. Para determinar si dentro de sus lugares de trabajo estas mujeres experimentaron formas de distinción y quizá de discriminación, se consultó sobre sus percepciones de diferenciación salarial y de trato con sus contrapartes latinos hombres, así como percepciones de trato desigual dentro de la organización en la cual prestan sus servicios. Los resultados presentados en el Gráfico 4 demuestran que la mayoría de estas mujeres (78\%) no percibió diferenciaciones en su entorno laboral y que el $91 \%$ de las encuestados no experimentó sentimientos de inferioridad. Al determinar las posibles razones de trato desigual, se muestra que las distinciones con porcentajes representativos son: una diferenciación por el tipo de trabajo que la encuestada realiza (78\%), es decir al ser mujeres líderes que imparten instrucciones y el tener un trato diferenciado por la forma de hablar y escribir el idioma inglés con $17 \%$, ya que dicho idioma no es su lengua materna.

GRÁFICO 4

ASPECTOS COMPROMETIDOS POR EL ÉXITO LABORAL MIGRATORIO

\begin{tabular}{|c|c|c|}
\hline Diferencia Salarial \& Trato - Sí & $21,7 \%$ & \\
\hline Diferencia Salarial \& Trato - No & & $78,3 \%$ \\
\hline Sentimientos Inferioridad en el trabajo - Sí & $8,7 \%$ & \\
\hline Sentimientos Inferioridad en el trabajo - No & & $91,3 \%$ \\
\hline Trato de desigualdad por trabajo realizado & & $78,3 \%$ \\
\hline Trato de desigualdad origen étnico & $4,3 \%$ & \\
\hline Trato de desigualdad manejo idioma Inglés & $17,4 \%$ & \\
\hline Trato de desigualdad por ser mujer & $8,7 \%$ & \\
\hline
\end{tabular}

Fuente: Encuesta Migración Calificada de Mujeres Latinas en el sur de la Florida, USA, 2014 Elaborado por: Maritza Figueroa

Fuente: Encuesta Migración Calificada de Mujeres Latinas en el sur de la Florida, USA, 2014

Elaborado por: Maritza Figueroa

Al consultar sobre las diferentes estrategias que dichas profesionales emplean o pensarían implementar para enfrentar los retos en el mercado labo- 
ral en los Estados Unidos de América, expresaron poner énfasis en la mejora de alguna habilidad (48\%) como el principal mecanismo para desarrollar su carrera profesional. El 45\% de las encuestadas desearía crear su propia empresa como otra de las estrategias para mantenerse activas en el mercado de trabajo. Se consultó además sobre los mecanismos que dichas profesionales podrían usar para posicionarse en el mercado laboral; un 50\% manifestó que harían uso de redes sociales virtuales para conseguir otras oportunidades laborales mientras y otro 50\% manifestó que contactarían a sus redes de familiares y amigos. Para más detalles ver Anexos 6 y 7.

Para conocer la relación entre el éxito migratorio y laboral, se consultó a las participantes sobre sus prioridades de vida. Un 35\% de encuestadas describió a su carrera profesional como un área de enfoque, un tercio de la población encuestada (30\%) manifestó que su matrimonio o vida de pareja era una prioridad y un 26\% expresó que por el momento desean vivir a plenitud las condiciones de vida que llevan en los Estados Unidos de América.

\section{CONCLUSIONES}

De la contextualización realizada, se puede concluir que la migración de mujeres latinas calificadas en ciertos aspectos se inscribe en las tendencias globales de acuerdo con lo que indica Coloma (2012) al analizar los datos del estudio de Lozano y Gandini (2009), donde el porcentaje de profesionales latinoamericanos que tienen un diploma universitario y que residen en los Estados Unidos de América es de 54,3\%. Al analizar a la población de este estudio, se establece que el $61 \%$ realiza actividades laborales acordes con su nivel de estudios, hallazgo que difiere de las tendencias de procesos de descualificación mostrados por
Koffman (2012), lo cual indicaría además que el sur del Estado de la Florida es un destino favorable para mujeres latinas calificadas. Así también, al considerar el tiempo de permanencia en destino, donde 17 de 23 de las encuestadas vivieron por un lapso de seis años o más, podemos ratificar lo que establece Lozano y Gandini (2009), donde la mayor cantidad de años de residencia en destino, en este caso en los Estados Unidos de América, influye para que los migrantes calificados puedan tener una mejor inserción laboral. Se demuestra además que existió una vinculación previa a organizaciones o estructuras fuera del Estado, nación 
que facilitaron su éxito laboral migratorio, donde 7 mujeres estaban vinculadas con sus empleadores por más de siete años y 5 de las encuestadas trabajaron de entre cuatro a seis años con la misma organización. Se demuestra también, que el contexto laboral en el que estas mujeres trabajan no genera formas de discriminación o sentimientos de subordinación, lo cual crea nuevas directrices para el análisis de la migración calificada femenina, donde el hecho de que una mujer latina migrante posea posiciones de liderazgo, no influye negativamente en su experiencia migratoria.

Al ser esta población un grupo de mujeres de origen latinoamericano, que poseen estudios superiores y donde la mayoría hace o haría uso de sus redes familiares y sociales (50\%) para acceder a otras formas de empleo, se demuestra claramente el efecto de interseccionalidad de clase, etnia y género como uno de los factores de logro personal y profesional de estas migrantes. Se demuestra también que muchas de estas mujeres son personas dedicadas a su profesión, es decir su trabajo es una prioridad fundamental de su vida; donde 17 de las encuestadas están casadas o tiene una pareja estable y 12 de ellas tienen hijos, lo cual indica que su trayectoria migratoria hizo posible el establecimiento de una familia y que son proveedoras de recursos en sus hogares.
Se concluye también que la migración calificada tiene un impacto positivo en mujeres latinas, lo que ratifica el planteamiento del Informe de Desarrollo Humano 2009 citado por Lozano y Gandini (2011), de no privar a las personas del derecho humano básico de desplazarse a otro país para alcanzar sus metas, ya que este proceso puede fomentar transformaciones que impactan positivamente a los migrantes.

Dado que literatura sobre migración calificada de mujeres de origen latinoamericano es casi nula, es necesario desarrollar análisis teóricos y estudios de caso sobre tendencias de migración calificada de individuos originarios de América Latina y describir rutas migratorias específicas de mujeres profesionales en mercados laborales foráneos. Así mismo, es importante realizar investigaciones relacionadas con prácticas y políticas migratorias para determinar aspectos de descualificación, equidad de género y discriminación, en relación con una población de mujeres latinas y profesionales. 


\section{BIBLIOGRAFÍA}

Coloma, S. (2012). "¿Qué tan distintos son los migrantes calificados? itinerarios migratorios: capital cultural y social entre migrantes ecuatorianos en Estados Unidos". (Tesis previa a la obtención de la maestría en Ciencias Sociales con mención en Sociología). Facultad Latinoamericana de Ciencias Sociales, FLACSO, Ecuador.

Ennls, S., Ríos-Vargas, Albert, N.G., (2012). La Población Hispana: 2010, Información del Censo del 2010. U.S. Department of Commerce Economics and Statistics Administration U.S. CENSUS BUREAU. Recuperado de: http://www.census.gov/ $\mathrm{prod} / \mathrm{cen} 2010 / \mathrm{briefs} / \mathrm{c} 2010 \mathrm{br}-$ 04sp.pdf (Visitado el 4 de agosto de 2014).

Dumont, J.C. et al. (2005). Labour Market Integration of Immigrant Women: Overview and Recent Trends, Paper prepared for the OECD and European Commission Seminar "Migrant Women and the Labor Market: Diversity and Challenges". Kofman, E. Gender and skilled migration in Europe. Cuadernos de Relaciones Laborales, 30(1) 2012. Universidad Complutense de Madrid.

Lozano, F. et al. (2009). La emigración de recursos humanos calificados desde países de América Latina y el
Caribe. Entre la fuga de cerebros y el desperdicio de talentos. Buenos Aires: XXVII Congreso de la Asociación Latinoamericana de Sociología.

Lozano, F. et al. (2011). Migración calificaday desarrollo humano en América Latina y el Caribe. Quito: Ponencia presentada en el IV Congreso de la Red Internacional de Migración y Desarrollo.

Riaño, I. et al. (2007). "Understanding the Labour Market Participation of Skilled Immigrant Women in Switzerland: The Interplay of Class, Ethnicity, and Gender" en nt. Migration \& Integration (2007) 8:163-183. Sandra Prasca. 
ReVista PUCE. ISSN 1390-7719.Núm, 103. 3 de mayo de 2016 -

ANEXO 1

PORCENTAJE POR PAÍS MUESTRA DEL ESTUDIO

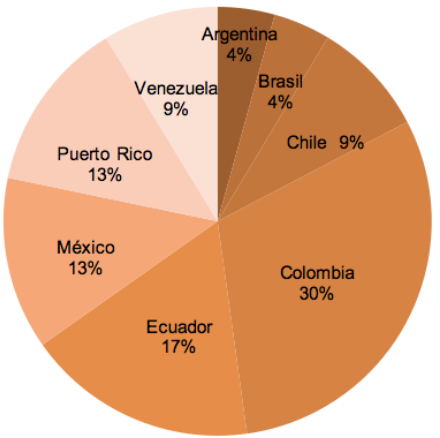

Fuente: Encuesta Migración Calificada de Mujeres Latinas en el sur de la Florida, USA, 2014

Elaborado por: Maritza Figueroa

ANEXO 2

PORCENTAJE POR EDAD MUESTRA DEL ESTUDIO

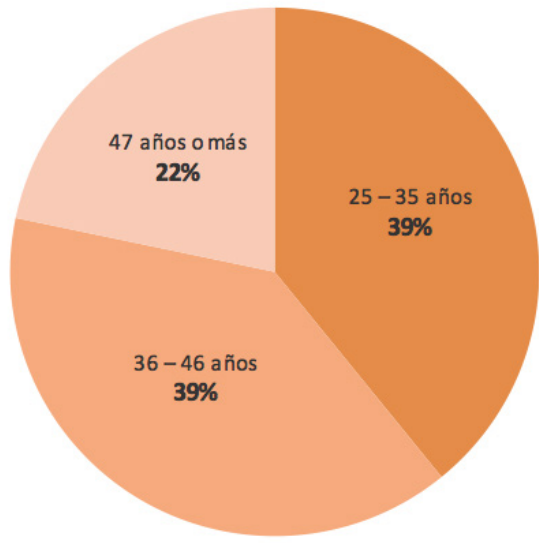

Fuente: Encuesta Migración Calificada de Mujeres Latinas en el sur de la Florida, USA, 2014

Elaborado por: Maritza Figueroa
ANEXO 3

PORCENTAJES NIVEL EDUCATIVO

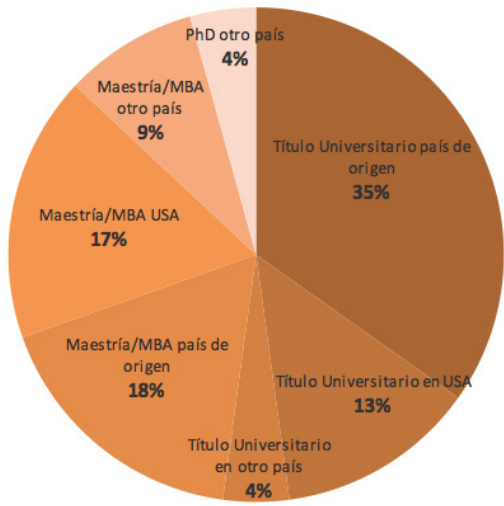

Fuente: Encuesta Migración Calificada de Mujeres Latinas en el sur de la Florida, USA, 2014

Elaborado por: Maritza Figueroa

\section{ANEXO 4}

TIPO DE RESIDENCIA / TIPO DE VISA EN EE.UU.

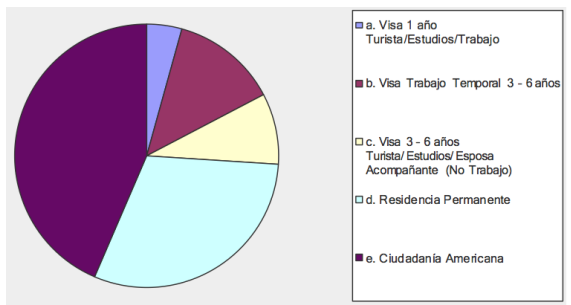

Fuente: Encuesta Migración Calificada de Mujeres Latinas en el sur de la Florida, USA, 2014

Elaborado por: Maritza Figueroa 
MUJERES LATINAS PROFESIONALES Y MIGRANTES CASOS DE ÉXITO

$\|$ ||\|I DE MUJERES CALIFICADAS EN EL SUR DE LA FLORIDA, EE. UU.

\section{ANEXO 5}

EXPECTATIVAS DE RUTA MIGRATORIA FUTURA

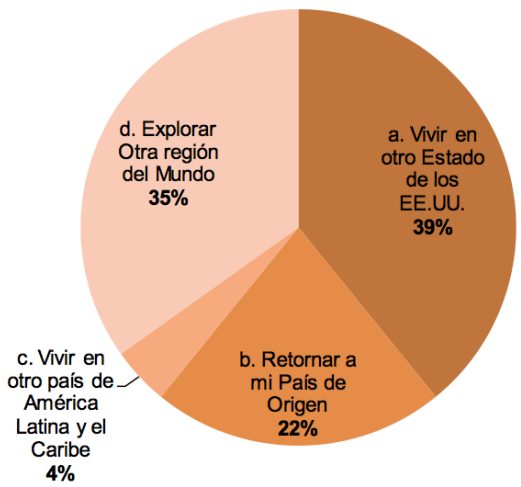

Fuente: Encuesta Migración Calificada de Mujeres Latinas en el sur de la Florida, USA, 2014

Elaborado por: Maritza Figueroa

\section{ANEXO 6}

ESTRATEGIAS PARA INSERCIÓN EN EL MERCADO LABORAL 1

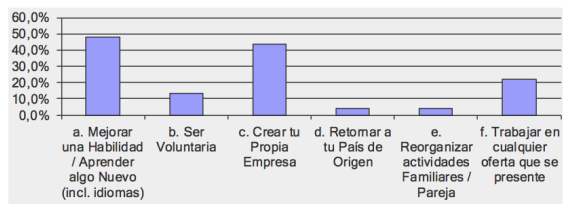

**Nota: esta pregunta permitía hacer la selección de más de una respuesta.

Fuente: Encuesta Migración Calificada de Mujeres Latinas en el sur de la Florida, USA, 2014

Elaborado por: Maritza Figueroa

\section{ANEXO 7}

ESTRATEGIAS PARA INSERCIÓN EN EL MERCADO LABORAL 2

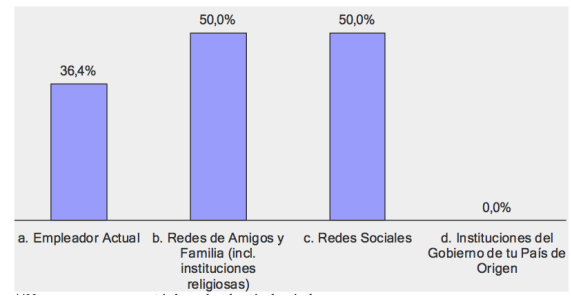

**Nota: esta pregunta permitía hacer la selección de más de una respuesta.

Fuente: Encuesta Migración Calificada de Mujeres Latinas en el sur de la Florida, USA, 2014

Elaborado por: Maritza Figueroa 